\title{
Displacement Damage in Bipolar Linear Integrated Circuits ${ }^{\dagger}$ \\ B. G. Rax A. H. Johnston and T. Miyahira \\ Jet Propulsion Laboratory \\ California Institute of Technology \\ Pasadena, California
}

\section{Introduction}

Although many different processes can be used to manufacture linear integrated circuits, the process that is used for most circuits is optimized for high voltage -- a total power supply voltage of about $40 \mathrm{~V}-$ and low cost. This process, which has changed little during the last twenty years, uses lateral and substrate pnp transistors. These pnp transistors have very wide base regions [1], increasing their sensitivity to displacement damage from electrons and protons. Although displacement damage effects can be easily treated for individual transistors $[2,3]$, the net effect on linear circuits can be far more complex because circuit operation often depends on the interaction of several internal transistors. Note also that some circuits are made with more advanced processes with much narrower base widths $[4,5]$. Devices fabricated with these newer processes are not expected to be significantly affected by displacement damage for proton fluences below $1 \times 10^{12} \mathrm{p} / \mathrm{cm}^{2}$.

This paper discusses displacement damage in linear integrated circuits with more complex failure modes than those exhibited by simpler devices, such as the LM1 11 comparator, where the dominant response mode is gain degradation of the input transistor [6]. Some circuits fail catastrophically at much lower equivalent total dose levels compared to tests with gamma rays. For example, Figure 1 shows test results for a radiationhardened op-amp with a JFET input stage. The device works satisfactorily up to nearly $1 \mathrm{Mrad}(\mathrm{Si})$ when it is irradiated with gamma rays, but fails catastrophically between 50 and $70 \mathrm{krad}(\mathrm{Si})$ when it is irradiated with protons.

\section{Experimental Approach and Circuit Technologies}

The devices in this study were irradiated at the cyclotron at the University of California, Davis, using $50 \mathrm{MeV}$ protons. The dose rate used for irradiation was approximately $40 \mathrm{rad}(\mathrm{Si}) / \mathrm{s}$, similar to dose rates often used for cobalt -60 irradiation of components. Electrical measurements were made with an LTS2020 integrated circuit test system and a Hewlett-Packard 4145 parameter analyzer.

the work described in this paper was carried out by the Jet Propulsion Laboratory, California Institute of Technology, under Propulsion Laboration. contract with the National Aeronautics and Space Adectronics Space Code AE. Work runded by the NASA

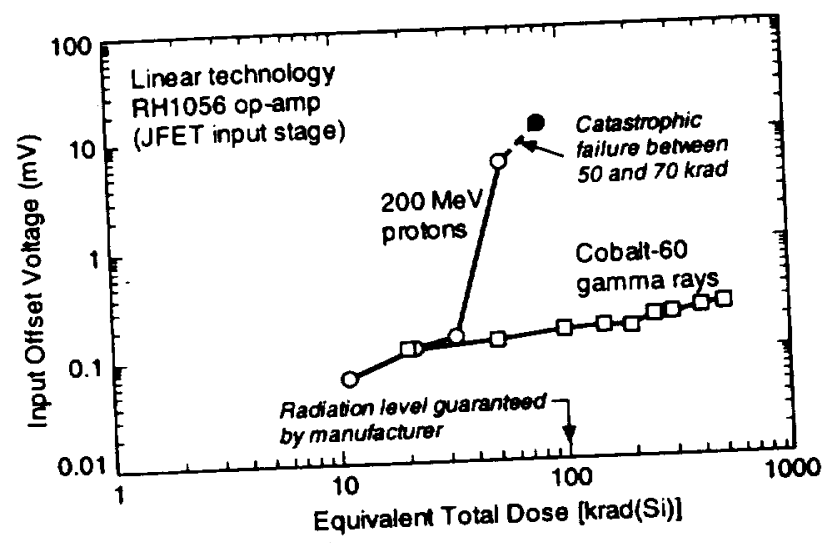

Figure 1. Degradation of the RH1056 op-amp from protons and gamma rays

The devices that were irradiated in the initial tests are shown in Table 1. They include hardened and unhardened versions of the LM137 negative voltage regulator, and hardened and unhardened versions of the OP27 op-amp. Both circuits use far more complex internal designs than the basic comparators and op-amps that are usually used to study degradation in linear devices. The OP27 has a compensated input stage, which uses a lateral pnp current source to provide a compensated (negative) input bias current that nearly cancels the positive input bias current required by the npn input transistor. Although this reduces the input bias current, it causes circuit operation to depend on the balance of this compensation scheme, which involves several different types of transistors.

Table 1. Devices Selected for Initial Proton Testing

\begin{tabular}{clll} 
Device & Function & Manuf. & Comments \\
\hline OP-27 & Op-amp & Anal. Dev. & \\
RH-27 & Op-amp & Lin. Tech. & Hard tech. \\
LM137 & Voltage reg. & National & \\
RH137 & Voltage reg. & Lin. Tech. & Hard tech.
\end{tabular}

Even though the hardened process from Linear Technology is designed to withstand ionization damage, the process uses lateral and substrate pnp transistors which are inherently sensitive to displacement damage because of the relatively wide base regions [2]. 


\section{Experimental Results}

Output voltage is one of the key parameters for voltage regulators, and most voltage regulators rely on bandgap reference circuits with npn transistors [7]. Figure 1 compares the output voltage degradation of the commercial and hardened LM137 voltage regulators. Both device types exhibit similar changes in that parameter. However, the commercial device stops functioning at about $25 \mathrm{krad}(\mathrm{Si})$; the failure is catastrophic and does not recovery even after extended time periods.

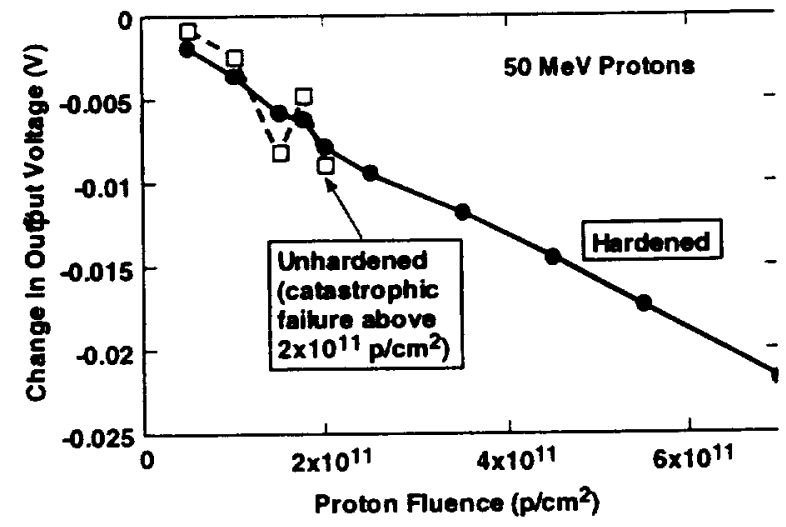

Figure 2. Effect of proton irradiation on output voltage degradation of hardened and commercial versions of the $\mathrm{LM} 137$ voltage regulator.

As shown in Figure 3, the unhardened LM137 devices continue to function with moderate output degradation up to $50 \mathrm{krad}(\mathrm{Si})$ when they are tested with cobalt-60 gamma rays / the devices actually functions at $100 \mathrm{krad}(\mathrm{Si})]$. However, a different failure mechanism comes into play when the devices are irradiated with protons which causes catastrophic failure. For the particular unit shown in Figure 3 catastrophic failure occurred between 24 and $28 \mathrm{krad}(\mathrm{Si})$; the shaded region shows the range of failure levels observed for 8 different units. Note that the spread in failure levels was about a factor of two.

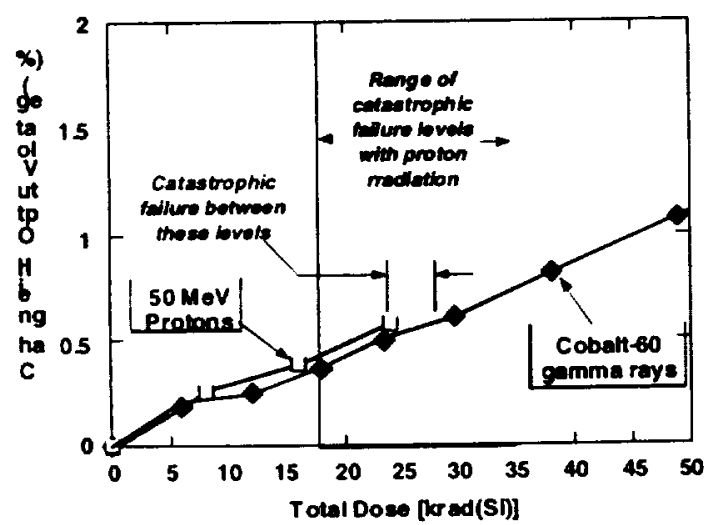

Figure 3. Change in output voltage of unhardened LMI 37 regulators when they are irradiated with protons and gamma rays.
Special input/output measurements were made to characterize this mechanism. Figure 4 shows how the transfer characteristics are affected by proton irradiation. The curve labeled "0" shows the preirradiation behavior. The output voltage begins to increase even at very low input voltages, but there is a slight nonlinearity at about $0.8 \mathrm{~V}$. This nonlinear region occurs when the start-up circuitry begins to operate. As the input voltage increases, the output voltage continues to increase until it reaches the cut-in voltage $(2.7 \mathrm{~V})$ at which point the output regulates to a very precise voltage level.

After the first irradiation level, the output voltage no longer responds to the input voltage until it reaches approximately $1.3 \mathrm{~V}$. At that point there is an abrupt increase in output voltage, and for voltages above that transition point the circuit behaves much like it did prior to irradiation. It continues to operate for input voltages above the cut-in voltage, with only about a $1 \%$ change in the regulated output voltage.

After the second irradiation level, the abrupt transition voltage condition has increased to $2.3 \mathrm{~V}$. The output voltage remains stuck at zero volts until the input voltage increases above that value, at which point the device begins to operate normally.

After the third irradiation (not shown on the figure) the device no longer functions, and the circuit will not operate even when the input voltage is raised to $40 \mathrm{~V}$. From these measurements, it is clear that the minimum input voltage required to cause the device to operate has increased to a level above the cut-in voltage, and the device has failed catastrophically

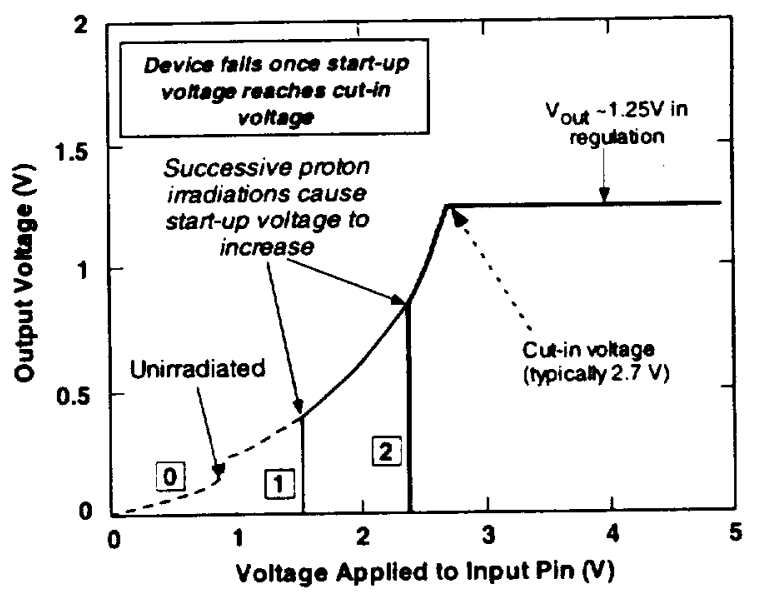

Figure 4. Input output characteristics of the LMI 37 voltage regulator before and after two proton irradiation levels.

A similar characterization method was used by Beaucour, et al., in a study of dose-rate effects of the LM137 [8]. However, there is an important difference in our results with protons. Beaucour, et al. showed that for ionization damage the devices that he studied would eventually start to regulate if the input voltage was 
increased beyond the "new" start-up voltage condition that occurred after irradiation. Consequently, the increase in start up voltage in their tests would only be important for applications with very low input/output voltage conditions. ${ }^{\dagger}$

The characteristics of the National LM137 were markedly different when they were irradiated with protons. After the start-up voltage degraded to the point where it exceeded the cut-in voltage, the device could not be made to operate even when the input voltage was raised to the maximum input voltage level. Thus, failure will occur even in applications with high input/output voltage "headroom."

The change of start-up conditions after various levels of proton irradiation are shown for several devices in

- Figure 5. The start-up voltage increases in a smooth, regular way as the radiation level increases. However, there are substantial differences in the radiation level at which catastrophic failure occurs. Note also that relatively small changes occurred when one of the devices was irradiated with gamma rays; the failure mode does not occur.

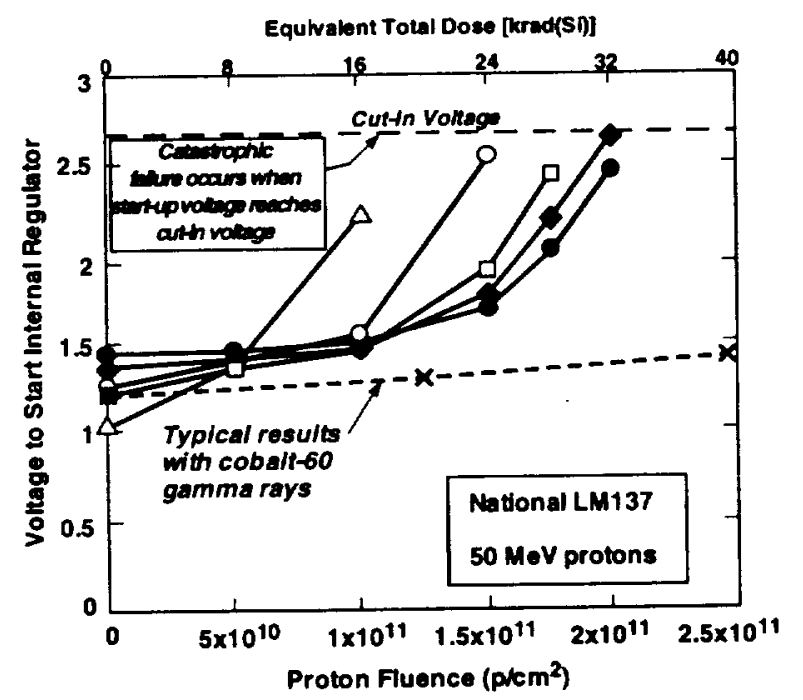

Figure 5. Dependence of start-up voltage on proton fluence for five devices illustrating the range of failure levels.

Proton tests were also done for the OP27 and RH27 op-amps. All of these devices continued to operate at high levels, even above $100 \mathrm{krad}(\mathrm{Si})$ [equivalent dose] when they were irradiated with protons. This is an interesting contrast with the RH1056 result, where a hardened device failed catastrophically at levels well below the design specification value.

$$
\text { -....... }
$$

†Beaucour, et al. did not identify the specific manufacturers of the LM 137 devices that they tested in their study. and it is possible that the devices for which the startup conditions changed were from a different manufacturer.
Note however that there are very large increases in input bias current for the commercial OP27 devices. These changes, which are always in the negative direction, occur because the lateral pnp compensation stage no longer operates properly. It overcompensates, causing very large negative input currents at both the inverting and noninverting inputs. However, other circuit parameters such as input offset voltage, openloop gain and output drive current are only slightly degraded. Note also that considerably more degradation occurs when the commercial devices are irradiated without bias compared to the results under bias. This suggests that ionization damage is the main reason for the degradation, not displacement damage.

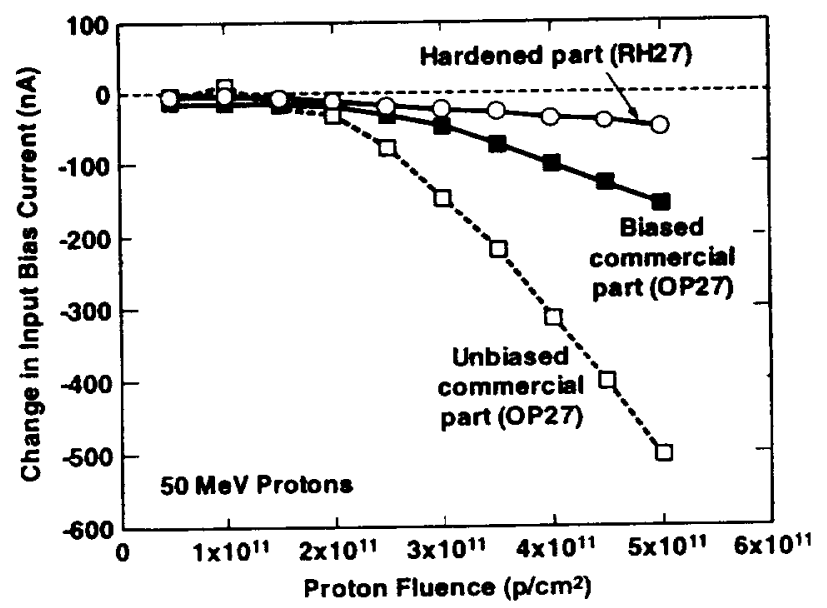

Figure 6. Degradation of input bias current of unhardened and hardened versions of the OP27 op-amp.

\section{Discussion}

Displacement damage will always cause more damage to occur from protons in wide-base pnp transistors compared to equivalent total dose levels from gamma rays. However, the net effect at the circuit level depends on the details of the circuit design. Many linear integrated circuits are designed to tolerate wide variations in the gain of internal pnp transistors, and for such circuits the increase in damage from displacement effects may be unimportant.

In cases where substantial degradation occurs from ionization damage (note the LMIII results from reference 6), the net impact of the increased displacement damage may be slight. However, for circuits like the RH 1056 and LM137, displacement damage causes catastrophic failure to occur, and the failure appears to be the result of failure mechanisms that are not present during tests in ionization environments, even when the tests are carried out at very high levels. This type of catastrophic failure is of extreme concern for space applications, and illustrates the need to test linear integrated circuits in proton environments. 
Note also that both ionization and displacement damage effects are significant for these circuits. Thus, it is not possible to do separate testing with neutrons to determine how displacement damage will combine with ionization damage at the circuit level.

Another potentially important factor is damage " linearity. Displacement damage continues to increase at successively higher proton radiation levels, but ionization damage for some processes (notably the National LMI11) saturates.

This work has shown that proton displacement damage can introduce different failure modes with catastrophic failure for some circuit types. Such effects can occur in both hardened and unhardened circuits. The reasons for such failures are complex, and depend on the internal design and margin for gain degradation.

- Catastrophic failure in voltage regulators is especially important because their failure can impact the operation of key subcircuit elements. It is important to recognize the importance of these effects and to include proton testing for certain types of linear integrated circuits.

\section{References}

I. R. S. Muller and T. I, Kamins, Device Electronics for Integrated Circuits, New York: John Wiley, 1986.

2. G. C. Messenger and M.S. Ash, The Effects of Radiation on Electronic Systems. New York: Van Nostrand Reinhold, 1992.

3. J. P. Raymond and E. L. Petersen, "Comparison of Neutron, Proton and Gamma Ray Effects in Semiconductor Devices," IEEE Trans. Nucl. Sci., 34, 1622 (1987).

4. J. Lapham, B. Scharf and R. Payne, "A Complementary Process for High Speed Precision Linear Circuits." Digest of Papers from the 1986 Bipolar Circuits and Technology Meeting, p. 31.

5. R. D. Schrimpf, et al., "Hardness Assurance Issues for Lateral PNP Bipolar Junction Transistors," IEEE Trans. Nucl. Sci., 42, 1641 (1995),

6. B. G. Rax, A. H. Johnston and C. I. Lee, "Proton Damage Effects in Linear Integrated Circuits," IEEE Trans. Nucl. Sci., $\underline{45}, 2632$ (1998).

7. B. G. Rax, C. I. Lee and A. H. Johnston, "Degradation of Precision Reference Devices in Space Environments,"

8. J. Beaucour, et al., "Total Dose Effects on Negative Voltage Regulator." IEEE Trans. Nucl. Sci., 41, 2420 (1994).

9. H. Barmaby, et al., "Analysis of Bipolar Linear Circuit Response Mechanisms for High and Low Dose Rate Irradiation," IEEE Trans. Nucl. Sci., 43, 3040 (1996). 